\title{
A hands-on approach to differential circuit measurements
}

\author{
R. Pallàs-Areny, O. Casas * \\ Department of Electronic Engineering, Instrumentation, Sensors and Interfaces Group, Castelldefels School of Technology, \\ Technical University of Catalonia, Avda. Canal Olímpic S/N 08860, Castelldefels, Barcelona, Spain
}

Received 11 May 2004; received in revised form 4 April 2006; accepted 9 May 2006

Available online 25 May 2006

\begin{abstract}
Electronic engineering is showing a definite trend towards differential circuits. However, most measurement instruments are single-ended. Consequently, engineering graduates often lack the skills to perform correct differential measurements. This paper describes the theoretical background, development and limitations of three experiments that help students to gain insight into differential measurements and the origin and consequences of a low common-mode rejection ratio. The experiments require only common equipment and suit both electrical and non-electrical engineering students.

(c) 2006 Elsevier Ltd. All rights reserved.
\end{abstract}

Keywords: Differential circuits; Differential measurements; Measurement teaching

\section{Introduction}

Differential circuits are increasingly common in both analog and digital electronics. Gone are the days when circuit designers would convert differential signals to single-ended signals at the very early stages of the measurement chain in order to further process them. Nowadays, because of the reduced cost of integrated circuits, differential circuits are not significantly more expensive than their single-ended counterparts. They also have interference-rejection capabilities and an increased linear dynamic range. This makes them very attractive for new designs. In fact, several commercial analog-to-digital converters

\footnotetext{
${ }^{*}$ Corresponding author. Tel.: +34 93413 7095; fax: +34 93413 7007.

E-mail addresses: ramon.pallas@upc.edu (R. Pallàs-Areny), jocp@eel.upc.edu (O. Casas).
}

(low-voltage models in particular), other analog signal processors and digital interface circuits have a differential input. However, measuring instruments, and oscilloscopes in particular, are mostly intended for single-ended measurements. As a result, many engineering graduates lack opportunities to acquire skills in differential measurements, in contrast with the availability of resources for simulating and designing differential circuits, either integrated [1] or based on discrete ICs [2].

This paper describes the theoretical background and three experiments that enable students to

(1) Understand the origin of the common-mode gain in differential circuits.

(2) Analyse the common-mode rejection ratio (CMRR) for cascaded differential stages.

(3) Realize that the CMRR is a complex quantity, not just a figure. 
The theoretical background helps in planning and performing the experiments and in analysing the results. The only materials needed are a common analog oscilloscope, two voltage probes and a sinusoidal voltage generator. The experiments are designed for senior undergraduate and graduate students in electrical engineering and graduate students in non-electrical engineering.

\section{Theoretical background and experiment planning}

According to Geddes [3], the differential amplifier was first used in the life sciences in around 1934. It was based on vacuum tubes, of course, and became quite popular because of its ability to reject powerline interference. Economy of components dictated that only the first stage should be differential. Circuit diagrams proposed by different researchers for biopotential recordings included some means of adjusting the gain of each input tube in order to make them equal, thus achieving a high CMRR. These circuits were based on push-pull amplifiers, which originated in 1929 in radio engineering as a means of increasing amplifier output power. Pushpull amplifiers were termed balanced or symmetrical amplifiers in the literature, but when the gain of the two amplifiers was very close (e.g., they differed by less than 1 part in 500), they were called differential amplifiers.

Differential amplifiers played a significant role as the basis for vacuum-tube-based operational amplifiers (op amps). This easily blurred their origin in the measurements field. Therefore, when transistors became available and the first textbooks were written, the teaching of differential circuits was based on the analysis of the emitter-coupled differential amplifier and related circuits (showing the formulas for the differential and common-mode gain) and the decomposition of the input voltage in differential and common-mode components [4]. Recent papers on CMOS design use the same approach [5]. However, a feasible alternative approach does not require transistor circuit analysis, so it may also interest non-electrical engineers.

Fig. 1 shows a differential circuit built from two single-ended amplifiers whose respective gains are $G_{\mathrm{A}}$ and $G_{\mathrm{B}}$.

Their respective output voltages can be obtained from

$$
\left.\begin{array}{l}
V_{\mathrm{A}}(j \omega)=G_{\mathrm{A}}(j \omega) V_{\mathrm{H}}(j \omega) \\
V_{\mathrm{B}}(j \omega)=G_{\mathrm{B}}(j \omega) V_{\mathrm{L}}(j \omega)
\end{array}\right\}
$$

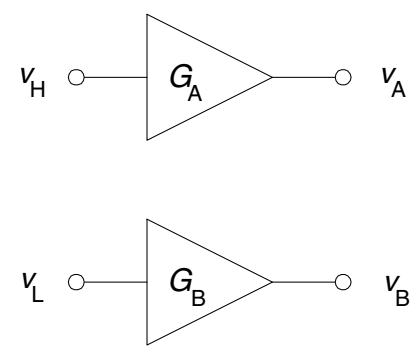

Fig. 1. Differential amplifier built from two single-ended amplifiers (for example, two channels of an oscilloscope that can display the difference between both channels' voltages).

and, writing the input voltages as a function of their differential and common-mode components,

$$
\left.\begin{array}{l}
v_{\mathrm{D}}=v_{\mathrm{H}}-v_{\mathrm{L}} \\
v_{\mathrm{C}}=\frac{v_{\mathrm{H}}+v_{\mathrm{L}}}{2}
\end{array}\right\}
$$

yields

$$
\left.\begin{array}{l}
V_{\mathrm{A}}(j \omega)=G_{\mathrm{A}}(j \omega) \frac{V_{\mathrm{D}}(j \omega)}{2}+G_{\mathrm{A}} V_{\mathrm{C}}(j \omega) \\
V_{\mathrm{B}}(j \omega)=-G_{\mathrm{B}}(j \omega) \frac{V_{\mathrm{D}}(j \omega)}{2}+G_{\mathrm{B}} V_{\mathrm{C}}(j \omega)
\end{array}\right\}
$$

Thus, the differential and common-mode output voltages are, respectively,

$$
\left.\begin{array}{c}
V_{\mathrm{A}}-V_{\mathrm{B}}=\frac{G_{\mathrm{A}}+G_{\mathrm{B}}}{2} V_{\mathrm{D}}+\left(G_{\mathrm{A}}-G_{\mathrm{B}}\right) V_{\mathrm{C}} \\
=G_{\mathrm{DD}} V_{\mathrm{D}}+G_{\mathrm{DC}} V_{\mathrm{C}} \\
\frac{V_{\mathrm{A}}+V_{\mathrm{B}}}{2}= \\
=\frac{G_{\mathrm{A}}-G_{\mathrm{B}}}{4} V_{\mathrm{D}}+\frac{G_{\mathrm{A}}+G_{\mathrm{B}}}{2} V_{\mathrm{C}} \\
=G_{\mathrm{CD}} V_{\mathrm{D}}+G_{\mathrm{CC}} V_{\mathrm{C}}
\end{array}\right\}
$$

where the frequency dependence has been obviated for the sake of simplicity and the definition of each of the four gains is implicit in the equations.

Therefore, the differential output signal is a result of both the differential and the common-mode input signal $\left(G_{\mathrm{DD}}, G_{\mathrm{DC}}\right)$. The same is true for the common-mode output signal $\left(G_{\mathrm{CD}}, G_{\mathrm{CC}}\right)$, but usually the ensuing stage is also differential and therefore designers are most concerned about the contribution of the common-mode input to the differential output. For the particular circuit in Fig. 1, $G_{\mathrm{DD}}=$ $G_{\mathrm{CC}}$. For a differential amplifier with single-ended output, $G_{\mathrm{DD}}=G_{\mathrm{D}}$ (differential gain) and $G_{\mathrm{DC}}=G_{\mathrm{C}}$ (common-mode gain). The CMRR is defined as

$\mathrm{CMRR}=\frac{G_{\mathrm{DD}}}{G_{\mathrm{DC}}}=\frac{1}{2} \frac{G_{\mathrm{A}}+G_{\mathrm{B}}}{G_{\mathrm{A}}-G_{\mathrm{B}}}=\frac{G}{\Delta G}$

The $G_{\mathrm{DD}} / G_{\mathrm{CC}}$ ratio is called the discrimination factor ( $\mathrm{D}$ in [2], $\mathrm{F}$ in [6]), but some authors call it the common-mode rejection ratio $\rho$ [7]. Here we prefer the CMRR definition based on Eq. (5) because it 
can also be applied to differential amplifiers with single-ended output.

This straightforward approach to differential circuit analysis shows that the common-mode gain arises from the mismatch between the two amplifiers. This can easily be verified through Experiment 1:

Step 1: Apply the same sinusoidal voltage to both vertical channels $(\mathrm{A}, \mathrm{B})$ of an oscilloscope using DC coupling and the same positions for the vertical channel attenuators.

Step 2: Superimpose both traces and, if necessary, use the gain/attenuator vernier to achieve good trace coincidence on the screen.

Step 3: Display A-B and calculate the CMRR at very low, low, medium and high frequencies and some intermediate frequencies (e.g., $10 \mathrm{~Hz}, 100 \mathrm{~Hz}, 1 \mathrm{kHz}, 10 \mathrm{kHz}, 100 \mathrm{kHz}$ and $1 \mathrm{MHz}$ ).

Step 4: Repeat this procedure with the vertical attenuators at "calibrated" settings and compare the results.

The phenomenon of channel mismatch resulting in a low CMRR can be further tested by using the AC-coupling feature of the oscilloscope. Oscilloscopes provide AC coupling by connecting a series DC-blocking capacitor to the front of the calibrated attenuator, whose equivalent impedance is $1 \mathrm{M} \Omega$, shunted by $15-45 \mathrm{pF}$. Fig. 2 shows the equivalent circuit, where the respective transfer functions for each input high-pass filter are $H_{\mathrm{A}}(j \omega)$ and $H_{\mathrm{B}}(j \omega)$.

By using the results in (4) and (5), we obtain

$$
\begin{aligned}
V_{\mathrm{A}}-V_{\mathrm{B}}= & \frac{H_{\mathrm{A}} G_{\mathrm{A}}+H_{\mathrm{B}} G_{\mathrm{B}}}{2} V_{\mathrm{D}} \\
& +\left(H_{\mathrm{A}} G_{\mathrm{A}}-H_{\mathrm{B}} G_{\mathrm{B}}\right) V_{\mathrm{C}} \\
\text { CMRR }= & \frac{1}{2} \frac{H_{\mathrm{A}} G_{\mathrm{A}}+H_{\mathrm{B}} G_{\mathrm{B}}}{H_{\mathrm{A}} G_{\mathrm{A}}-H_{\mathrm{B}} G_{\mathrm{B}}}
\end{aligned}
$$

By defining the average of and difference between the transfer functions of the respective input filters for each channel, as follows:

$$
\left.\begin{array}{l}
H=\frac{H_{\mathrm{A}}+H_{\mathrm{B}}}{2} \\
\Delta H=H_{\mathrm{A}}-H_{\mathrm{B}}
\end{array}\right\}
$$

and by replacing $H_{\mathrm{A}}$ and $H_{\mathrm{B}}$ in (7), we have

$$
\begin{aligned}
\frac{1}{\mathrm{CMRR}} & =\frac{2 H\left(G_{\mathrm{A}}-G_{\mathrm{B}}\right)+\Delta H\left(G_{\mathrm{A}}+G_{\mathrm{B}}\right)}{H\left(G_{\mathrm{A}}+G_{\mathrm{B}}\right)+\frac{\Delta H}{2}\left(G_{\mathrm{A}}-G_{\mathrm{B}}\right)} \\
& \cong \frac{\Delta G}{G}+\frac{\Delta H}{H}=\frac{1}{\mathrm{CMRR}_{\mathrm{G}}}+\frac{1}{\mathrm{CMRR}_{\mathrm{H}}}
\end{aligned}
$$
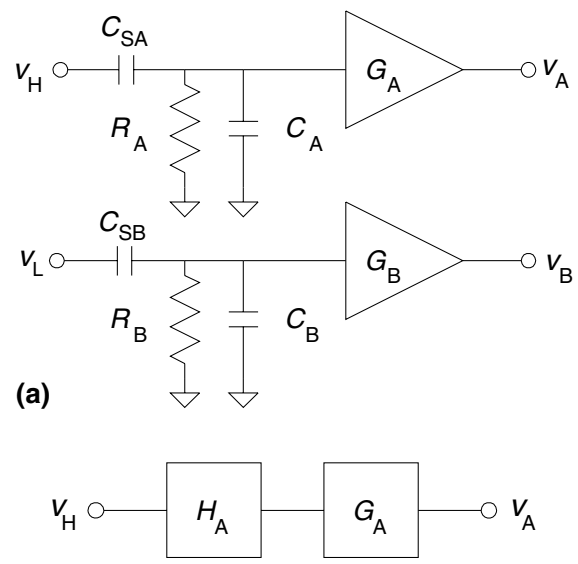

(b)

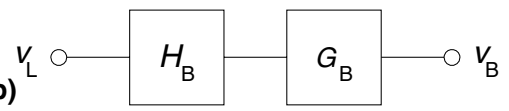

Fig. 2. (a) Equivalent circuit for the two AC-coupled vertical channels of an oscilloscope, and (b) their block diagram.

where the approximation holds whenever $\Delta H\left(G_{\mathrm{A}}-\right.$ $\left.G_{\mathrm{B}}\right) / 2 \ll H\left(G_{\mathrm{A}}+G_{\mathrm{B}}\right)$, that is, when $4 \times \mathrm{CMRR}_{\mathrm{G}} \times$ $\mathrm{CMRR}_{\mathrm{H}} \gg 1$, which is often the case.

Eq. (9) shows that the imbalance of both the input filters $(H)$ and the attenuators/amplifiers $(G)$ degrades the overall CMRR. In the absence of $C_{\mathrm{A}}$ and $C_{\mathrm{B}}, \mathrm{CMRR}_{\mathrm{H}}$ would be finite only at frequencies relatively close to the respective corner frequency of each high-pass filter. $C_{\mathrm{A}}$ and $C_{\mathrm{B}}$, however, degrade $\mathrm{CMRR}_{\mathrm{H}}$ at high frequencies. Experiment 2 illustrates some of these effects:

Step 1: Start from Step 2 in Experiment 1 and switch both oscilloscope channels to AC coupling.

Step 2: Display A-B and calculate the CMRR at very low, low, medium and high frequencies and some intermediate frequencies (e.g., $10 \mathrm{~Hz}, 100 \mathrm{~Hz}, 1 \mathrm{kHz}, 10 \mathrm{kHz}, 100 \mathrm{kHz}$ and $1 \mathrm{MHz}$ ). The minimal frequency should be close to the corner frequency of the ACcoupling circuit.

Step 3: Compare CMRR to $\mathrm{CMRR}_{\mathrm{G}}$ (measured in Experiment 1).

Step 4: With the $1 \mathrm{kHz}$ sinusoidal voltage applied to both channels, try to reduce the trace amplitude by adjusting the gain/attenuator vernier. 
Eq. (9) also shows that if $\mathrm{CMRR}_{\mathrm{G}}=-\mathrm{CMRR}_{\mathrm{H}}$, the CMRR would be infinite. Because the CMRR is a complex quantity (the ratio between two frequency-dependent gains), this condition cannot be achieved for all frequencies. Moreover, the component values in Fig. 2(a) cannot be modified, so $\mathrm{CMRR}_{\mathrm{H}}$ is determined by component tolerances and trimming is impossible. This shows the importance of the balance between the two channels in a differential circuit built from two separate singleended circuits.

To illustrate the effects of unbalanced circuits and show that they can arise even when good design practices are applied for single-ended circuits, consider voltage probes with a 10:1 voltage attenuation ratio. Fig. 3 shows the equivalent circuit for each voltage probe connected to a DC-coupled oscilloscope channel at frequencies low enough to eliminate stray inductances.

Alternatively, the adjustable capacitor can be placed in parallel with the input oscilloscope capacitance, but the objective and final results are the same: the capacitor is adjusted until $R_{\mathrm{A}} C_{\mathrm{A}}=R_{\mathrm{PA}} C_{\mathrm{PA}}=$ $\tau_{\mathrm{A}}$. When the probe has been properly compensated, all frequencies undergo the same attenuation and we have

$H_{\mathrm{A}}=\frac{Z_{\mathrm{A}}}{Z_{\mathrm{A}}+Z_{\mathrm{PA}}}=\frac{R_{\mathrm{A}}}{R_{\mathrm{A}}+R_{\mathrm{PA}}}=k_{\mathrm{A}}$

Similarly, when the probe of channel $B$ is compensated,

$H_{\mathrm{B}}=\frac{Z_{\mathrm{B}}}{Z_{\mathrm{B}}+Z_{\mathrm{PB}}}=\frac{R_{\mathrm{B}}}{R_{\mathrm{B}}+R_{\mathrm{PB}}}=k_{\mathrm{B}}$

The equivalent input impedance for each signal channel, including a compensated voltage probe, is then

$Z_{\mathrm{CA}}=\frac{1}{j \omega C_{\mathrm{AI}}}+\frac{R_{\mathrm{A}}+R_{\mathrm{PA}}}{1+j \omega R_{\mathrm{A}} C_{\mathrm{A}}}$

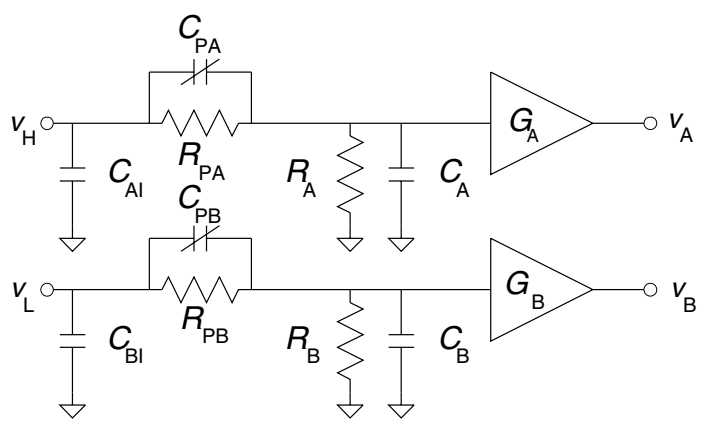

Fig. 3. Simplified model of two oscilloscope voltage probes connected to the vertical channels of an oscilloscope.
$Z_{\mathrm{CB}}=\frac{1}{j \omega C_{\mathrm{BI}}}+\frac{R_{\mathrm{B}}+R_{\mathrm{PB}}}{1+j \omega R_{\mathrm{B}} C_{\mathrm{B}}}$

where $C_{\mathrm{AI}}$ and $C_{\mathrm{BI}}$ are the respective stray capacitances of each probe tip $(<1 \mathrm{pF})$, which cannot be compensated by the adjustable capacitor. Each input signal undergoes the attenuation-amplification process shown in Fig. 4. The resulting voltages are

$$
\left.\begin{array}{l}
V_{\mathrm{A}}=V_{\mathrm{H}} \frac{Z_{\mathrm{CA}}}{Z_{\mathrm{HO}}+Z_{\mathrm{CA}}} k_{\mathrm{A}} G_{\mathrm{A}} \\
V_{\mathrm{B}}=V_{\mathrm{L}} \frac{Z_{\mathrm{CB}}}{Z_{\mathrm{LO}}+Z_{\mathrm{CB}}} k_{\mathrm{B}} G_{\mathrm{B}}
\end{array}\right\}
$$

If the same voltage source is applied to both voltage probes, their outputs may be different because of their different input impedance, voltage attenuation $(k)$ or oscilloscope gain $(G)$. Therefore, these are the three factors that can degrade the overall CMRR.

Experiment 3 assesses the importance of $k_{\mathrm{A}} \neq k_{\mathrm{B}}$ and highlights that voltage probe compensation cannot ensure good differential measurements:

Step 1: Use the "calibration" signal from the oscilloscope to compensate each of two voltage probes. (Common oscilloscopes have a square voltage of $1 \mathrm{kHz}$.)

Step 2: Apply the same sinusoidal voltage to both vertical channels $(\mathrm{A}, \mathrm{B})$ of the oscilloscope using DC coupling, the same vertical attenuator positions and the two compensated voltage probes.

Step 3: Superimpose the two traces and, if necessary, use the gain/attenuator vernier to achieve good trace coincidence on the screen.
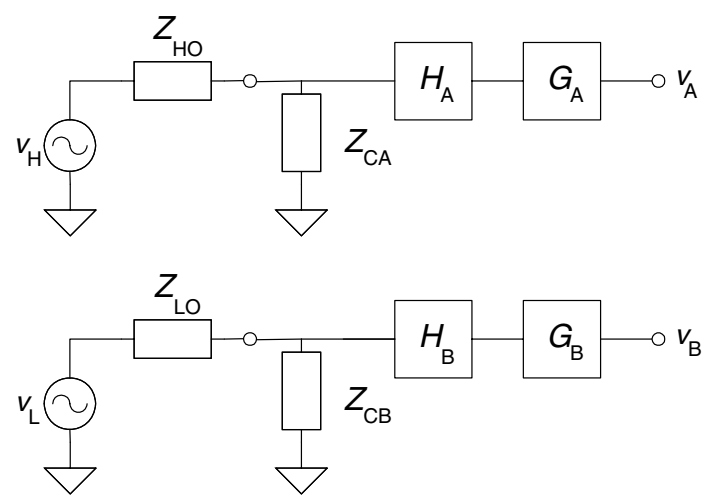

Fig. 4. Model of the signal source, voltage probe and oscilloscope system. 
Step 4: Display A-B and calculate the CMRR at very low, low, medium and high frequencies (e.g., $10 \mathrm{~Hz}, 100 \mathrm{~Hz}, 10 \mathrm{kHz}$ and $1 \mathrm{MHz}$ ).

Step 5: Repeat the procedure at $1 \mathrm{kHz}$, but "adjust" one of the voltage probes again until an output of zero is obtained (i.e., a horizontal trace on the screen).

\section{Experiments and discussion}

We performed the three experiments described above using the following equipment and materials:

(1) OD-512 analog oscilloscope (20 MHz, $Z_{\mathrm{A}}, Z_{\mathrm{B}}$ : $1 \mathrm{M} \Omega \| 25 \mathrm{pF}$, low cost), manufactured by Promax Electrónica (L'Hospitalet de Llobregat, Spain)

(2) RG58 coaxial cable $(1.5 \mathrm{~m})$.

(3) HZ-36 voltage probes, manufactured by Hameg (Frankfurt, Germany). These probes have a switch that allows them to be used as $1: 1$ or $10: 1$ attenuators $(10 \mathrm{MHz} / 100 \mathrm{MHz}$ bandwidth).

(3) GF-232 pulse/function generator (2 MHz, $50 \Omega$, single-ended, ground-referred output), manufactured by Promax Electrónica.

One end of the coaxial cable was connected to the output of the function generator and the other end was connected to the two voltage probes.

\section{Experiment 1:}

Step 1: We applied a $1 \mathrm{kHz}, 20 \mathrm{~V}$ peak-to-peak sinusoidal voltage to both vertical channels $(\mathrm{A}, \mathrm{B})$ of the oscilloscope using the voltage probes in the 1:1 position. The oscilloscope was DC-coupled and both vertical attenuators were at $5 \mathrm{~V} / \mathrm{div}$.

Step 2: When the two signals were superimposed, their traces on the screen coincided. There was no need to adjust the vernier control.

Step 3: We switched to A-B display mode, which yielded an almost horizontal trace (meaning $0 \mathrm{~V}$ ). However, by switching each vertical attenuator to $1 \mathrm{~V} /$ div, we obtained a sine wave with a peak-to-peak amplitude of about $0.2 \mathrm{~V}$. By switching the vertical attenuators to more sensitive positions, we obtained a distorted signal. Hence, back in the $1 \mathrm{~V} /$ div position, we obtained |CMRR $(1 \mathrm{kHz}) \mid=40 \mathrm{~dB}$. The first row of Table 1 shows the results at other frequencies.
Because of the lack of memory (or persistence), measurements below $50 \mathrm{~Hz}$ were impractical. The measurement resolution was about $0.2 \mathrm{~V} / 10=0.02 \mathrm{~V}$. Therefore, for a $20 \mathrm{~V}$ input, measurements by a different person may give CMRR results that differ by about $1 \mathrm{~dB}[20 \mathrm{lg}(20 / 0.2)$ vs. $20 \mathrm{lg}$ $(20 / 0.18)]$.

Step 4: Using the vernier control of both channels, we reduced the displayed $0.2 \mathrm{~V}$ signal to an amplitude below the resolution at $100 \mathrm{~Hz}, 1 \mathrm{kHz}$ and $10 \mathrm{kHz}$. At $100 \mathrm{kHz}$ and $1 \mathrm{MHz}$, we were able to reduce the amplitude, but not as much. We discerned the minimal output voltage by noticing that, when one of the gains was continuously adjusted, a phase inversion in the signal was displayed. The minimal voltage was obtained before the phase inversion started. Fast sweep rates were better than slow sweep rates for perceiving small amplitudes; the visual resolution was improved by displaying a minimum of ten cycles. For $100 \mathrm{~Hz}, 1 \mathrm{kHz}$ and $10 \mathrm{kHz}$, the knob positions that yielded the minimal output were approximately the same. To obtain a minimal output at $100 \mathrm{kHz}$ and $1 \mathrm{MHz}$, one of the vernier knobs had to be readjusted. The signal displayed was very sensitive to the knob settings and the resolution of the potentiometer reduced the repeatability of the results.

The results in Step 2 mean that $G_{\mathrm{A}}=G_{\mathrm{B}}$ when measured with the available resolution in the $5 \mathrm{~V} /$ div attenuator position, which can be estimated as one tenth of a division (width of the trace on the screen), that is, $0.5 \mathrm{~V}$. Step 3, however, shows that: (1) by improving the resolution to $0.1 \mathrm{~V}$, we were able to detect the difference between $G_{\mathrm{A}}$ and $G_{\mathrm{B}}$, and (2) the improvement in resolution was limited by output distortion due to the common-mode voltage (the voltage applied to both channels), even if the differential signal (difference signal for the deflection amplifiers) was quite small. As a result, the maximum CMRR that we were able to measure with this oscilloscope was limited to $20 \mathrm{~V} / 0.1 \mathrm{~V}=$ $46 \mathrm{~dB}$, which was certainly quite small. The first row of Table 1 shows that the dissimilarity between the two vertical attenuators of the oscilloscope remained constant up to at least $1 \mathrm{MHz}$. In order to test what happens near $20 \mathrm{MHz}$, we needed a 
Table 1

Sample of CMRR measurements obtained with a low-cost analog oscilloscope in different measurement conditions

\begin{tabular}{llllll}
\hline CMRR (dB) & Setup & Freq. & & & \\
\cline { 3 - 6 } & & $100 \mathrm{~Hz}$ & $1 \mathrm{kHz}$ & $10 \mathrm{kHz}$ & $100 \mathrm{kHz}$ \\
\hline Experiment 1 & DC coupling & 40 & 40 & 40 & 40 \\
Experiment 2 & AC coupling & 40 & 40 & 40 & 40 \\
Experiment 3 & AC-DC coupling & 28 & 40 & 40 & 40 \\
\hline
\end{tabular}

function generator with higher output frequency. Step 4 shows that adjusting $G_{\mathrm{A}}$ and $G_{\mathrm{B}}$ until $G_{\mathrm{A}}=G_{\mathrm{B}}$ improved the CMRR, as expected from (5). However, at high frequencies, where the phase shift of each amplifier becomes large, we were unable to achieve the condition $G_{\mathrm{A}}=G_{\mathrm{B}}$.

\section{Experiment 2:}

Step 1: Starting from Step 2 in Experiment 1, we switched both oscilloscope channels to AC coupling.

Step 2: When displaying A-B, the CMRR was in the second row in Table 1. According to the manufacturer, the corner frequency of the AC-coupling filter was below $10 \mathrm{~Hz}$, but very slow signals could not be appropriately displayed with this oscilloscope.

Step 3: At $100 \mathrm{~Hz}$, the CMRR did not change because of the AC-coupling filters. This means that the corner frequency of the filters was under $100 \mathrm{~Hz}$. The low measurement resolution prevented us from perceiving the effect of different corner frequencies for each channel. However, the third row in Table 1 shows the effect of switching one channel to DC. The CMRR dropped to just $28 \mathrm{~dB}$ at $100 \mathrm{~Hz}$, as expected from (9).

Step 4: With a sinusoidal voltage of $1 \mathrm{kHz}$ applied to both channels, we adjusted the gain/ attenuator vernier until the differential output was close to $0 \mathrm{~V}$. We also obtained an output close to $0 \mathrm{~V}$ at $100 \mathrm{~Hz}$ and $10 \mathrm{kHz}$ and a minimal output at $100 \mathrm{kHz}$ and $1 \mathrm{MHz}$. However, the vernier knobs had to be readjusted for the higher frequencies.

\section{Experiment 3:}

Step 1: With both voltage probes set at 10:1 attenuation, we separately compensated each probe using the square voltage of $1 \mathrm{kHz}$ from the oscilloscope.
Step 2: We applied a $20 \mathrm{~V}$ peak-to-peak sine voltage to both DC-coupled vertical channels, which were set at the $0.5 \mathrm{~V} / \mathrm{div}$ position (because the actual voltage at the input of each vertical channel was $2 \mathrm{~V}$ ).

Step 3: We superimposed the two traces and the signals coincided. Therefore, the attenuation of each probe was the same as if it were assessed with a $0.5 \mathrm{~V}$ resolution.

Step 4: We switched both vertical attenuators to $0.1 \mathrm{~V} /$ div and displayed A-B. This yielded $20 \mathrm{mV}$ from $100 \mathrm{~Hz}$ to $100 \mathrm{kHz}$ and $40 \mathrm{mV}$ at $1 \mathrm{MHz}$. Row four of Table 1 shows the CMRR calculated. Because $Z_{\mathrm{CA}}, Z_{\mathrm{CB}} \gg$ $50 \Omega$, the reduced CMRR was probably a result of $k_{\mathrm{A}} \neq k_{\mathrm{B}}$.

Step 5: By readjusting one of the voltage probes, we were able to obtain a minimal voltage at $1 \mathrm{kHz}$. We displayed the calibration signal using the readjusted probe, which revealed that the probe was no longer compensated. This explains why voltage probes for differential measurements have two adjustments: one to compensate each probe separately and another to achieve $k_{\mathrm{A}}=k_{\mathrm{B}}$. For voltage probes with a single adjustment, a slight variation in $k$ has a slight effect on the compensation but a strong effect on the CMRR.

\section{Summary}

Differential measurements performed with singleended instruments whose outputs are subtracted require balanced channels. In common single-ended oscilloscopes, channel matching can easily be worse than 1 in 100, which limits the CMRR to about $40 \mathrm{~dB}$. Voltage probes do not improve differential measurements. In fact, they may reduce the overall CMRR. AC coupling can also contribute to channel mismatch. Overall, the low CMRR of single-ended oscilloscopes limits them greatly for differential measurements, such as AC-bridge output detection. 
The closeness of the results in Table 1 depends on the particular oscilloscope used. Here, very low-cost instruments were used in order to show that the experiments do not require any special equipment. However, in some teaching environments, this closeness may reduce student interest in the experiments. As an alternative, in Experiment 3, a $1 \mathrm{k} \Omega$ resistor can be placed in series with the output of the function generator in order to increase the effect of the low input impedance. Under- or over-compensating one of the voltage divider probes would also worsen the CMRR. Advanced students may also find it instructive to derive the expression for the overall CMRR of the voltage-probe-oscilloscope system from (12).

\section{Acknowledgements}

This project was funded by the Spanish Ministry of Science and Technology, project DPI2002-00707. The authors also appreciate the help of graduate students Isabel Fernández and Pablo Cotaimich in repeating the experiments.

\section{References}

[1] P.R. Gray, R.G. Meyer, Analysis and design of analog integrated circuits, John Wiley \& Sons, New York, 2001.

[2] R. Pallàs-Areny, J.G. Webster, Analog Signal Processing, John Wiley \& Sons, New York, 1999.

[3] L.A. Geddes, Who invented the differential amplifier? IEEE Engineering in Medicine and Biology Magazine 15 (3) (1996) $116-117$

[4] N.R. Malik, Teaching difference amplifier concepts, IEEE Transactions on Education 27 (1) (1984) 26-30.

[5] F. Corsi, C. Marzocca, An approach to the analysis of the CMOS differential stage with active load and single-ended output, IEEE Transactions on Education 46 (3) (2003) 325328.

[6] A.F.P. van Putten, Electronic Measurement Systems, Prentice-Hall, New York, 1988.

[7] S.A. Witherspoon, J. Choma Jr., The analysis of balanced linear differential circuits, IEEE Transactions on Education 38 (1) (1995) 40-50. 